Check for updates

New York

Cite this as: BMJ 2021;375:n3098 http://dx.doi.org/10.1136/bmj.n3098 Published: 16 December 2021

\section{Covid 19: US cases rise amid omicron fears but booster shots offer protection, experts say}

\author{
Janice Hopkins Tanne
}

Some 800 ooo Americans have died from covid-19, the highest number of any country in the world, and cases, deaths, hospital admissions, and local transmission are increasing as the threat of omicron is growing, Rochelle Walensky, director of the Centers for Disease Control and Prevention (CDC), has said.

However, two doses of an mRNA vaccine and a booster provide good protection and there is no need for a booster specific for the variant, said Anthony Fauci, director of the National Institute of Allergy and Infectious Diseases and President Joe Biden's medical advisor. Both spoke at a White House press briefing yesterday on 15 December. ${ }^{1}$

Jeffrey Zients, the White House coronavirus response coordinator, said, "The US vaccination programme has already prevented 10.3 million hospital admissions. And it has saved 1.1 million American lives."

Although most US cases are caused by the delta variant, Walensky said, "We expect to see the proportion of omicron cases here in the US continue to grow in the coming weeks. Early data suggest that omicron is more transmissible than delta, with a doubling time of about two days." Omicron is now causing about $3 \%$ of new cases nationally, but about $13 \%$ in the north eastern states of New Jersey and New York.

Walensky said there are about 118 ooo new cases of covid-19 every day and about 1100 deaths. The CDC predicted that covid-19 deaths would rise to between 821000 and 837000 by 1 January $2022 .{ }^{2}$ She said it was vital for everyone to get vaccinated and receive a booster shot if they are eligible. Some 200 million of the US population of 330 million have been vaccinated and about 55 million have received boosters. Vaccination is available to everyone aged 5 and over.

Walensky said that substantial or high community transmission is now occurring in about $90 \%$ of the nation's 3142 counties and she advised people to wear masks in public indoor settings.

Vaccination, boosting, and masking are especially critical for the most vulnerable people, including seniors, pregnant people, and those who are immunocompromised, she said. Nursing home residents who are fully vaccinated and have received a booster dose have a 10 times lower rate of getting covid-19 compared with residents who have only received a primary vaccination series or are unvaccinated.

Fauci said that boosters were essential in light of the omicron variant. In vitro studies showed a substantial drop in neutralising antibodies about three weeks after the second dose of the Pfizer BioNTech vaccine. In people who had received two doses of an mRNA vaccine plus a booster dose there was, however, "a substantial increase in neutralising activity.” This occurred with both the Pfizer and the Moderna vaccines in studies at the National Institute of Health, he said. The increase after the third dose was "well within the range of neutralising omicron," he said.

Fauci said that although the omicron variant compromises the effectiveness of the two dose mRNA vaccination and reduces overall protection, it still protects against severe disease.

"At this point, there is no need for a variant specific booster. The message remains clear: if you are unvaccinated, get vaccinated. And particularly in the arena of omicron, if you are fully vaccinated, get your booster shot.”

1 Press briefing by White House covid- $\mathbb{1 9}$ response team and public health officials. 15 December 2021. www.whitehouse.gov/briefing-room/pressbriefings/2021/12/15/press-briefing-by-white-house-covid-19-responseteam-and-public-health-officials-73.

2 Centers for Disease Control and Prevention. Covid-19 forecasts: deaths. 15 December 2021. www.cdc. gov/coronavirus/2019-ncov/science/forecasting/forecasting-us.html. 